\title{
Characteristics and risk factors associated with missed diagnosis in patients with smear-negative pulmonary tuberculosis
}

\author{
Se Hyun Kwak, Ji Soo Choi, Eun Hye Lee, Su Hwan Lee, Ah Young Leem, Sang Hoon Lee, Song Yee Kim, \\ Kyung Soo Chung, Ji Ye Jung, Moo Suk Park, Young Sam Kim, Joon Chang, and Young Ae Kang
}

Division of Pulmonology, Department of Internal Medicine, Severance Hospital, Yonsei University College of Medicine, Seoul, Korea

Received: December 20, 2019 Revised : March 31, 2020 Accepted: April 8, 2020

\section{Correspondence to}

Young Ae Kang, M.D.

Division of Pulmonology,

Department of Internal

Medicine, Severance Hospital,

Yonsei University College

of Medicine, 50-1 Yonsei-ro,

Seodaemun-gu, Seoul 03722,

Korea

Tel: $+82-2-2228-1954$

Fax: +82-2-393-6884

E-mail: mdkang@yuhs.ac

https:/orcid.org/0000-0002-

7783-5271
Background/Aims: Delayed diagnosis and treatment of smear-negative pulmonary tuberculosis (TB) are major concerns for TB control. We evaluated characteristics of patients with smear-negative pulmonary TB who received a delayed diagnosis and identified risk factors that may have contributed to this delay.

Methods: We reviewed medical records of patients with smear-negative culturepositive pulmonary TB treated at a tertiary care hospital in South Korea between January 2017 and December 2018. Patients who initiated anti-TB treatment after positive cultures were included in the missed TB group, and those who initiated empirical treatment before positive cultures were included in the control group.

Results: Of 220 patients included, 117 (53.2\%) and 103 (46.8\%) were in the missed TB and control groups, respectively. Patients in the missed TB group were older $(p$ $=0.001)$ and had a higher mean body mass index $(\mathrm{BMI})(p=0.019)$. Comorbidities (66.9\% vs. $46.6 \%, p=0.003$ ) and immunocompromised patients $(33.1 \%$ vs. $20.4 \%$, $p=0.035)$ were more common in the missed TB group than in the control group. Old age (odds ratio [OR], 1.030; 95\% confidence interval [CI], 1.012 to $1.048 ; p=$ 0.001 ), high BMI (OR, 1.114; 95\% CI, 1.004 to 1.237; $p=0.042$ ), and negative polymerase chain reaction (PCR) results (OR, 9.551; 95\% CI, 4.925 to 18.521; $p<0.001$ ) were associated with delayed diagnosis.

Conclusions: In more than half of patients with smear-negative pulmonary TB, the diagnosis was delayed. Patients with delayed TB diagnosis were older, had higher BMI, and negative PCR results.

Keywords: Lung disease; Tuberculosis; Diagnosis; Risk factors

\section{INTRODUCTION}

Tuberculosis (T'B) is the leading cause of death from a single epidemic disease worldwide [1], and it remains a global health problem despite advances in its diagnosis and treatment. The global incidence of TB is currently declining $1.5 \%$ per year [1], and to achieve the World Health Organization (WHO) End TB Strategy targets, this reduction rate needs to be increased by $4 \%$ to $5 \%$ per year by 2020 [2].

To reach the goals of the WHO End TB Strategy, early diagnosis to prevent TB spread is one of the most important factors for reducing the incidence of TB [3]. To enable early detection and diagnosis, the sensitivity and specificity of TB diagnostic tools must be improved. Sputum smear microscopy has been used as a primary 
method for diagnosing TB [4]; however, the sensitivity of this method is reportedly only $50 \%$ to $60 \%$ [5].

Because it may take a long time before TB culture test results are confirmed, not initiating TB treatment until a positive culture report is received may result in delayed diagnosis. This, in turn, may delay optimal treatment, lead to poor treatment outcomes, and increase the risk of transmission in the hospital and community. For the rapid diagnosis of smear-negative pulmonary TB, TB polymerase chain reaction (PCR), which includes the Xpert MTB/RIF assay (Xpert, Cepheid, Sunnyvale, CA, USA), is performed; however, this assay is not sufficiently sensitive and not fully implemented in real-world settings. Therefore, delayed diagnosis and treatment of smear-negative pulmonary TB are major concerns for TB control in low- and intermediate-burden areas.

We, therefore, aimed to evaluate the clinical characteristics and radiologic findings of patients with smear-negative pulmonary TB whose diagnosis and treatment were delayed and to identify risk factors that may have contributed to the delayed diagnosis.

\section{METHODS}

\section{Study population and design}

We conducted this single-center retrospective study at a tertiary care hospital in South Korea between January 2017 and December 2018. Patients with smear-negative and culture-positive pulmonary TB were retrospectively identified. The Institutional Review Board at Severance Hospital approved this retrospective study and waived the need for informed consent for access to electric medical records (IRB No. 2019-2828-001).

\section{Patients and data collection}

Medical records of 307 adult patients (aged $\geq 18$ years) with smear-negative and culture-positive pulmonary TB were evaluated. Smears were considered positive if acid-fast bacteria (on auramine-rhodamine staining) were observed in the smears of any of the respiratory specimens [6]. We included only patients diagnosed with pulmonary TB according to the WHO [7] and Korean TB guidelines [8], i.e., a diagnosis that was based on a positive culture from a spontaneous sputum sample, an induced sputum sample, or bronchoalveolar lavage.
From this patient group, we excluded patients with extra-pulmonary TB, those who had already initiated treatment at another hospital, those who were not prescribed medication at our hospital, and those who were currently under treatment.

The following data were collected from each patient's medical record: demographic data (sex, age, body mass index [BMI]), presence of comorbidities (immunocompromised condition, hypertension, diabetes mellitus, chronic kidney disease, and respiratory disease), smoking status, and prior history of TB treatment. Immunocompromised patients were defined as (1) hematopoietic cell transplant recipients; (2) other solid organ transplant recipients; (3) patients with malignancies who were receiving chemotherapy; and (4) patients who received any immunosuppressive treatments (e.g., biologic agents targeting inflammatory mediators, or corticosteroid therapy). Patients who smoked at least once in their lifetime were classified as smokers. Furthermore, we reviewed other medical records, including clinical T'B forms, reports of symptoms at diagnosis, radiographic findings, and diagnostic methods.

\section{Classification of the study population}

The study population was divided into two groups based on whether anti-TB treatment was initiated before or after receiving positive culture results. Patients who initiated anti-TB treatment after the confirmation of positive culture results were included in the missed TB group, and those who initiated empirical treatment before obtaining the final culture results were included in the control group.

\section{Treatment outcomes}

Treatment outcomes were defined according to the WHO [9] and Korean TB guidelines [8]. A favorable outcome was defined as a cure and/or treatment completion without relapse, and an unfavorable outcome was defined as treatment failure, death during treatment, or relapse after completing the initial treatment.

\section{Statistical analysis}

Data are presented as the number of cases and the median (interquartile range [IQR]). Categorical comparisons were performed using the chi-square test, and continuous variables were compared using the Mann-Whitney 
test or $t$ test. Multivariate logistic regression analysis was performed to evaluate factors potentially associated with a delayed diagnosis of TB. The odds ratios (ORs) and $95 \%$ confidence intervals (CIs) were also calculated. A $p$ value $<0.05$ was considered significant for all analyses. Data analysis was performed using SPSS version 25 (Released 2017, IBM Corp., Armonk, NY, USA).

\section{RESULTS}

\section{Study population}

From January 2017 to December 2018, 307 patients with smear-negative and culture-positive TB were enrolled in this study. Of the 307 patients, 87 were excluded, and 220 patients met the inclusion criteria. Of these 220 patients, $117(53.2 \%)$ were included in the missed TB group and 103 (46.8\%) were included in the control group (Fig. 1).

\section{Baseline characteristics}

Baseline characteristics of the study population are summarized in Table 1. The study population had a median age of 58.0 years (IQR, 39.3 to 71.0). Compared with patients in the control group, those in the missed TB group were older (median age, 49.0 years [IQR, 33.0 to 69.0] vs. 63.0 years [IQR, 48.5 to 72.5]; $p=0.001$ ) and had significantly higher BMI $\left(21.6 \mathrm{~kg} / \mathrm{m}^{2}\right.$ [IQR, 19.5 to 23.4] vs. $22.4 \mathrm{~kg} / \mathrm{m}^{2}$ [IQR, 20.0 to 24.7], $p=0.019$ ). The presence of any comorbidities (79 [66.9\%] vs. 48 [46.6\%], $p=0.003$ ) and immunocompromised conditions (39 [33.1\%] vs. 21

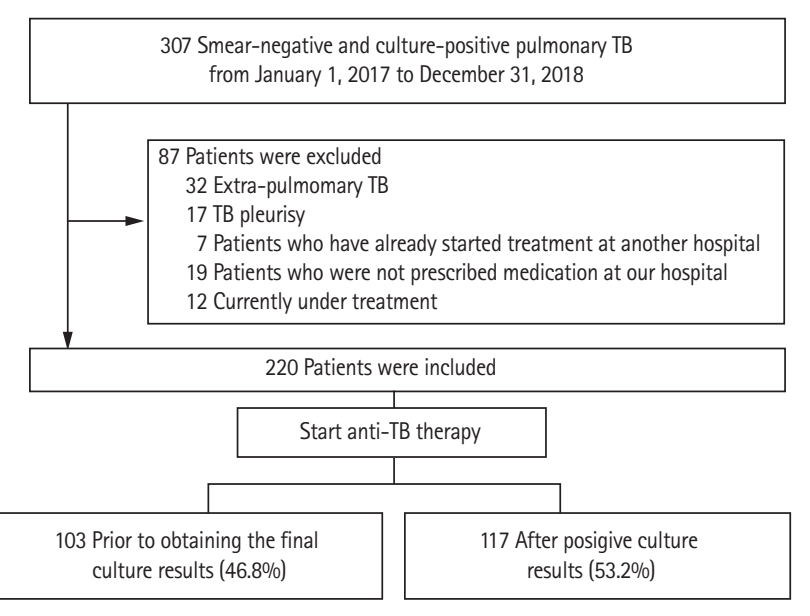

Figure 1. Flow chart of the study population. TB, tuberculosis.
[20.4\%], $p=0.035)$ were more common in the missed T'B group than in the control group. There were no significant differences in sex, smoking status, or prior history of TB treatment between the two groups.

The most common symptoms experienced by patients before being diagnosed with TB were cough and sputum production (64 [29.1\%] and 43 [19.5\%], respectively). There were no significant differences between the two groups regarding whether symptoms developed before TB was diagnosed (52 [50.5\%] vs. 64 [54.7\%], $p=0.589)$. In total, 216 (98.2\%) patients underwent chest computed tomography (CT), and four (1.8\%) underwent only chest X-ray. Fewer patients in the missed TB group than in the control group presented with a cavitating lesion on chest X-ray or chest CT (24 [23.3\%] vs. 12 [10.3\%], $p=0.011)$. In addition to the smear/culture examination, patients underwent other diagnostic tests, including PCR and interferon-gamma release assay (IGRA). In total, 30.9\% of patients underwent IGRA, of whom $95.6 \%$ showed positive results. There were no significant differences between the two groups in terms of the proportion of patients with positive IGRA results (37 [97.4\%] vs. 28 [93.3\%], $p=0.056$ ). Furthermore, $92.3 \%$ of patients underwent PCR, including Xpert MTB/RIF assay, and $47.8 \%$ of patients with positive cultures also had positive PCR results. The proportion of positive PCR results was lower in the missed TB group than in the control group (26 [25.5\%] vs. 71 [70.3\%], $p<0.001$ ) (Table 1 and Fig. 2).

Table 2 shows working diagnoses for patients in the missed TB group before pulmonary TB was confirmed and the reasons for initiating anti-TB treatment before obtaining culture results for patients in the control group. Clinicians considered the possibility of TB at the first visit in $71(60.7 \%)$ patients. Of these 71 patients, 27 (38.0\%) were treated with antibiotics before the diagnosis of TB to rule out pulmonary TB. Differential diagnoses considered by clinicians for patients in the missed TB group included pneumonia $(\mathrm{n}=24,20.5 \%)$ and lung cancer $(n=8,6.8 \%)$. Patients who initiated anti-TB treatment before obtaining culture results were treated based on positive PCR results ( $\mathrm{n}=48,46.6 \%$ ), radiographic findings $(\mathrm{n}=27,26.2 \%)$, radiographic findings and PCR ( $n=20,19.4 \%)$, pathology results $(n=4,3.9 \%)$, pathology and $\operatorname{PCR}(n=3,2.9 \%)$, or clinical symptoms $(n=1,1.0 \%)$. 
Table 1. Baseline characteristics

\begin{tabular}{|c|c|c|c|c|}
\hline Variable & $\operatorname{Total}(\mathrm{n}=\mathbf{2 2 0})$ & Control $(\mathrm{n}=103)$ & Missed TB $(\mathrm{n}=117)$ & $p$ value \\
\hline Age, yr & $58.0(39.3-71.0)$ & $49.0(33.0-69.0)$ & $63.0(48.5-72.5)$ & 0.001 \\
\hline Sex & & & & 1.000 \\
\hline Male & $130(59.1)$ & $61(59.2)$ & $69(59.0)$ & \\
\hline Female & $90(40.9)$ & $42(40.8)$ & $48(41.0)$ & \\
\hline Body mass index, $\mathrm{kg} / \mathrm{m}^{2}$ & $22.0(19.7-24.1)$ & $21.6(19.5-23.4)$ & $22.4(20.0-24.7)$ & 0.019 \\
\hline \multicolumn{5}{|l|}{ Underlying disease } \\
\hline Presence of any comorbidity & $127(57 \cdot 7)$ & $48(46.6)$ & $79(67 \cdot 5)$ & 0.003 \\
\hline Immunocompromised & $60(27 \cdot 3)$ & $21(20.4)$ & $39(33 \cdot 3)$ & 0.035 \\
\hline Hypertension & $58(26.4)$ & $25(24 \cdot 3)$ & $33(28.2)$ & 0.542 \\
\hline Diabetes mellitus & $39(17.7)$ & $15(14.6)$ & $24(20.5)$ & 0.290 \\
\hline Chronic kidney disease & $11(5.0)$ & $4(3 \cdot 9)$ & $7(6.0)$ & 0.548 \\
\hline Respiratory disease & $13(5 \cdot 9)$ & $3(2.9)$ & $10(8.5)$ & 0.091 \\
\hline Smoking history & $103(46.8)$ & $47(45 \cdot 6)$ & $56(47 \cdot 9)$ & 0.787 \\
\hline Prior history of TB treatment & $29(13.2)$ & $12(11.7)$ & $17(14 \cdot 5)$ & 0.556 \\
\hline Symptoms & $116(52.7)$ & $52(50.5)$ & $64(54.7)$ & 0.589 \\
\hline Cough & $64(29.1)$ & $31(30.1)$ & $33(28.2)$ & 0.768 \\
\hline Sputum & $43(19 \cdot 5)$ & $14(13 \cdot 6)$ & $29(24.8)$ & 0.042 \\
\hline Night sweat & $1(0.5)$ & $1(1.0)$ & $0(0.0)$ & 0.468 \\
\hline Fever & $30(13.6)$ & $13(12.6)$ & $17(14 \cdot 5)$ & 0.699 \\
\hline Weight loss & $2(0.9)$ & $0(0.0)$ & $2(1.7)$ & 0.500 \\
\hline Dyspnea & $16(7 \cdot 3)$ & $6(5.8)$ & $10(8.5)$ & 0.604 \\
\hline Hemoptysis & $12(5 \cdot 5)$ & $6(5.8)$ & $6(5 \cdot 1)$ & 1.000 \\
\hline \multicolumn{5}{|l|}{ Radiographic finding $^{\mathrm{a}}$} \\
\hline Cavitary lesion & $36(16.4)$ & $24(23 \cdot 3)$ & $12(10.3)$ & 0.011 \\
\hline Consolidation & $82(37 \cdot 3)$ & $43(41.7)$ & $39(33 \cdot 3)$ & 0.211 \\
\hline Nodules/mass & $180(81.8)$ & $88(85 \cdot 4)$ & $92(78.6)$ & 0.222 \\
\hline Predominant upper lung field & $107(48.6)$ & $55(53 \cdot 4)$ & $52(44 \cdot 4)$ & 0.224 \\
\hline \multicolumn{5}{|l|}{ IGRA } \\
\hline Done & $68(30.9)$ & $38(36.9)$ & $30(25.6)$ & 0.080 \\
\hline Positive & $65(95.6)$ & $37(97 \cdot 4)$ & $28(93.3)$ & 0.056 \\
\hline \multicolumn{5}{|l|}{ PCR } \\
\hline Done & $203(92.3)$ & $101(98.1)$ & $102(87.2)$ & 0.002 \\
\hline Positive & $97(47.8)$ & $71(70.3)$ & $26(25 \cdot 5)$ & $<0.001$ \\
\hline Bronchoscopy & $55(25 \cdot 0)$ & $28(27.2)$ & $27(23.1)$ & 0.534 \\
\hline Considering the possibility of TB at first visit & $170(77 \cdot 3)$ & $99(96.1)$ & $71(60.7)$ & $<0.001$ \\
\hline
\end{tabular}

Values are presented as median (interquartile range) or number (\%).

TB, tuberculosis; IGRA, interferon-gamma release assay; PCR, polymerase chain reaction.

${ }^{a}$ Chest computed tomographies (CTs) were performed in 216 patients. We reviewed all chest CTs in 216 patients and chest $\mathrm{X}$-rays in another four patients. 


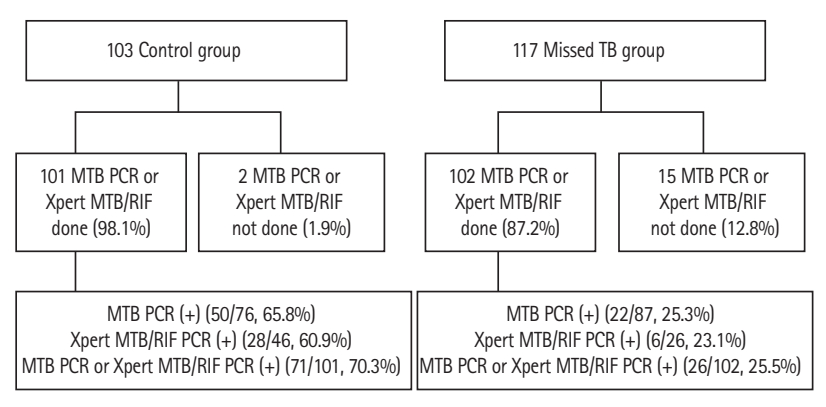

Figure 2. Proportion of positive polymerase chain reaction (PCR), including Xpert MTB/RIF assay (Xpert, Cepheid, Sunnyvale, CA, USA) and results.

\section{Clinical treatment outcomes}

Clinical treatment outcomes are shown in Table 3. The median duration from the date of culture testing to the start of treatment was 3.0 days (IQR, 0.0 to 7.0) in the control group and 25.0 days (IQR, 21.0 to 29.0) in the missed TB group $(p<0.001)$, indicating that anti-TB treatment was initiated significantly later in the missed TB group than in the control group (Table 3 and Fig. 3). During the median follow-up of 559.0 days (IQR, 311.0 to 767.0 ), 190 (86.4\%) patients were treated successfully with favorable outcomes, including cure and treatment completion. Unfavorable outcomes occurred in 12 patients (5.5\%); these outcomes included treatment failure $(n=1,0.5 \%)$, death during treatment $(\mathrm{n}=10,4.5 \%)$, and relapse after initial treatment completion $(\mathrm{n}=1,0.5 \%)$. The median duration of treatment was 188.0 days (IQR, 181.0 to 269.0). There were no significant differences between the two groups in the median duration of treatment (188.0 days [IQR, 181.0 to 271.o] vs. 186.0 days [IQR, 181.0 to 267.5], p $=0.893$ ). Eighty-one (36.8\%) patients experienced adverse events associated with anti-TB drugs, of whom 17 (7.7\%) changed medication and 38 (17.3\%) discontinued and restarted medication owing to the adverse events. There were no statistically significant differences in treatment outcomes between the two groups.

\section{Factors associated with a delayed diagnosis of TB}

Table 4 shows the results of univariate and multivariate logistic regression analyses that estimated the ORs of missed TB. On univariate analysis, six variables were associated with missed TB diagnosis, namely, old age, high BMI, immunocompromised status, absence of a cavitating lesion, absence of sputum production, and
Table 2. Working diagnoses in the missed TB group and the reasons for starting anti-TB treatment before obtaining the culture results in the control group

\begin{tabular}{|cc}
\hline Value & No. (\%) \\
\hline Working diagnoses $^{\mathrm{a}}$ & \\
\hline TB suspected in the first impression & $71 / 117(60.7)$ \\
\hline Pneumonia & $24 / 117(20.5)$ \\
\hline Lung cancer & $8 / 117(6.8)$ \\
\hline Stabilized TB & $5 / 117(4.3)$ \\
\hline NTM & $5 / 117(4.3)$ \\
\hline Bronchiectasis & $2 / 117(1.7)$ \\
\hline Latent TB & $1 / 117(0.9)$ \\
\hline IgG4 related systemic disease & $1 / 117(0.9)$ \\
\hline Reasons for starting anti-TB therapy & \\
\hline Clinical symptoms only & $1 / 103(1.0)$ \\
\hline Radiographic findings only & $27 / 103(26.2)$ \\
\hline Radiographic findings and PCR & $20 / 103(19.4)$ \\
\hline PCR & $48 / 103(46.6)$ \\
\hline Pathology & $4 / 103(3.9)$ \\
\hline Pathology and PCR & $3 / 103(2.9)$ \\
\hline
\end{tabular}

TB, tuberculosis; NTM, nontuberculosis mycobacteria; $\mathrm{IgG}_{4}$, immunoglobulin $\mathrm{G}_{4}$; PCR, polymerase chain reaction.

${ }^{a}$ Working diagnoses in missed TB group.

${ }^{b}$ Reasons for starting anti-TB therapy before positive culture in control group.

negative PCR results or no PCR testing. The following variables remained significant in the final multivariate analysis: older age (OR, 1.030; 95\% CI, 1.012 to 1.048; $p$ $=0.001)$, high BMI (OR, 1.114; $95 \%$ CI, 1.004 to $1.237 ; p=$ 0.042), and negative PCR results (OR, 9.551; 95\% CI, 4.925 to $18.521 ; p<0.001$ ).

\section{DISCUSSION}

In this study, we found that the diagnosis of more than half of all patients with smear-negative pulmonary TB (53.2\%) was delayed until the confirmation of positive culture results. Such a delay could contribute to disease transmission within the hospital [10] and community. Patients with a delayed diagnosis of TB were older, had higher BMIs, and were more likely to have negative PCR results. Thus, these were factors that could potentially 
Table 3. Clinical treatment outcomes

\begin{tabular}{|c|c|c|c|c|}
\hline Variable & $\operatorname{Total}(\mathrm{n}=220)$ & $\begin{array}{l}\text { Control } \\
(\mathrm{n}=103)\end{array}$ & $\begin{array}{l}\text { Missed TB } \\
(\mathrm{n}=117)\end{array}$ & $p$ value \\
\hline $\begin{array}{l}\text { Period between culture test and the start of } \\
\text { treatment, day }\end{array}$ & $17.0(4.0-26.0)$ & $3.0(0.0-7.0)$ & $25.0(21.0-29.0)$ & $<0.001$ \\
\hline Treatment duration, day & $188.0(181.0-269.0)$ & $188.0(181.0-271.0)$ & $186.0(181.0-267.5)$ & 0.893 \\
\hline End of treatment outcome & & & & 0.923 \\
\hline Treatment success & $190(86.4)$ & $89(86.4)$ & $101(86.3)$ & \\
\hline Failure & $1(0.5)$ & o & $1(0.9)$ & \\
\hline Death during treatment & $10(4 \cdot 5)$ & $4(3 \cdot 9)$ & $6(5.1)$ & \\
\hline Lost to follow-up & $2(0.9)$ & $1(1.0)$ & $1(0.9)$ & \\
\hline Not evaluated & $17(7 \cdot 7)$ & $9(8.7)$ & $8(6.8)$ & \\
\hline \multicolumn{5}{|l|}{ Treatment outcomes in follow-up } \\
\hline Relapse & $1(0.5)$ & o & $1(0.9)$ & 1.000 \\
\hline All-cause death & $11(5 \cdot 0)$ & $4(3 \cdot 9)$ & $7(6.0)$ & 0.548 \\
\hline Favorable outcome & $189(85.9)$ & $89(86.4)$ & $100(85.5)$ & 1.000 \\
\hline Unfavorable outcome & $12(5 \cdot 5)$ & $4(3 \cdot 9)$ & $8(6.8)$ & 0.386 \\
\hline Changed medication due to $\mathrm{AE}$ & $17(7 \cdot 7)$ & $7(6.8)$ & $10(8.5)$ & 0.801 \\
\hline Stopped medication due to $\mathrm{AE}$ & $38(17 \cdot 3)$ & $15(14 \cdot 6)$ & $23(19 \cdot 7)$ & 0.373 \\
\hline Total of patient who have AE & $81(36.8)$ & $35(34 \cdot 0)$ & $46(39 \cdot 3)$ & 0.484 \\
\hline Time of follow-up, day & $559.0(311.0-767.0)$ & $559.0(306.0-762.0)$ & $561.0(318.0-775.0)$ & 0.272 \\
\hline
\end{tabular}

Values are presented as median (interquartile range) or number (\%).

TB, tuberculosis; AE, adverse events.

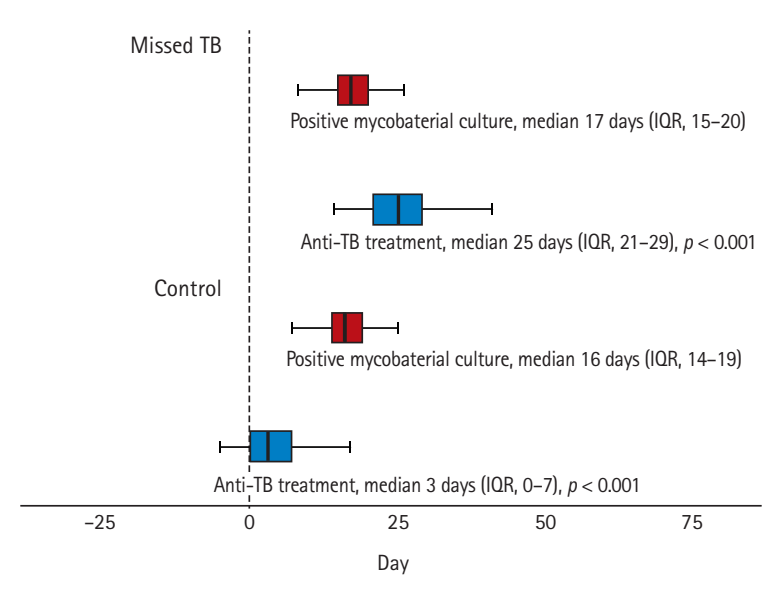

Figure 3. Box-and-whisker plots showing the date of initiation of anti-tuberculosis (TB) treatment and positive results of mycobacterial culture. IQR, interquartile range.

delay diagnosis and treatment of smear-negative pulmonary TB.

Older age was an important factor associated with missed TB diagnosis, a finding consistent with the re- sults of previous studies [11-13]. In elderly people, clinical symptoms and radiographic findings of TB are known to be less typical and less specific [12,14], making it easier for TB to be confused with other diseases [13]. Atypical clinical manifestations of TB in elderly patients may lead to delayed diagnosis and treatment, which may be associated with poor treatment outcomes. Furthermore, because adverse reactions are more common in elderly patients undergoing anti-TB treatment $[15,16]$, clinicians are more likely to hesitate to initiate anti-TB treatment, which can cause a delay in the diagnosis and treatment of such patients with smear-negative pulmonary TB. Previous studies have shown that the mortality rate of TB increases with older age [17]. The higher mortality rate associated with TB in elderly patients was because of underlying comorbidities, deterioration of the immune system, and delays in initiating anti-TB treatment [18]. Therefore, efforts to improve early detection and treatment in the elderly population are needed. TB is often difficult to diagnose; thus, even if typical respiratory symptoms of TB are not present, clinicians 
Table 4. Univariate and multivariate logistic regression analysis of variables that may have contributed to a missed TB diagnosis

\begin{tabular}{|c|c|c|c|c|c|c|}
\hline \multirow{2}{*}{ Variable } & \multicolumn{3}{|c|}{ Unadjusted } & \multicolumn{3}{|c|}{ Adjusted } \\
\hline & OR & $95 \% \mathrm{CI}$ & $p$ value & OR & $95 \% \mathrm{CI}$ & $p$ value \\
\hline Old age & 1.027 & $1.012-1.042$ & $<0.001$ & 1.030 & $1.012-1.048$ & 0.001 \\
\hline Male sex & 0.990 & $0.578-1.696$ & 0.970 & 0.882 & $0.445^{-1.711}$ & 0.882 \\
\hline Body mass index & 1.109 & $1.014-1.213$ & 0.023 & 1.114 & $1.004-1.237$ & 0.042 \\
\hline \multicolumn{7}{|l|}{ Underlying disease } \\
\hline Immunocompromised & 1.952 & $1.056-3.609$ & 0.033 & 1.258 & $0.600-2.637$ & 0.544 \\
\hline Hypertension & 1.226 & $0.670-2.243$ & 0.509 & & & \\
\hline Diabetes mellitus & 1.514 & $0.746-3.073$ & 0.251 & & & \\
\hline Chronic kidney disease & 1.575 & $0.448-5.542$ & 0.479 & & & \\
\hline Respiratory disease & 3.115 & $0.833-11.647$ & 0.091 & & & \\
\hline Smoking history & 1.094 & $0.643-1.860$ & 0.741 & & & \\
\hline Prior history of TB treatment & 1.289 & $0.584-2.845$ & 0.529 & & & \\
\hline \multicolumn{7}{|l|}{ Symptoms } \\
\hline Without sputum & 4.77 & $0.236-0.964$ & 0.039 & 0.605 & $0.263-1.392$ & 0.237 \\
\hline \multicolumn{7}{|l|}{ Radiographic findings } \\
\hline Without cavitary lesion & 2.658 & $1.253-5.638$ & 0.011 & 1.747 & $0.716-4.260$ & 0.220 \\
\hline Without consolidation & 1.433 & $0.828-2.481$ & 0.198 & & & \\
\hline Without nodules/mass & 1.594 & $0.789-3.222$ & 0.194 & & & \\
\hline Without pleural effusion & 1.137 & $0.070-18.415$ & 0.928 & & & \\
\hline Non-predominant upper lung field & 1.432 & $0.842-2.438$ & 0.185 & & & \\
\hline PCR, negative or not done & $7 \cdot 766$ & $4.247-14.198$ & $<0.001$ & $9 \cdot 55^{1}$ & $4.925^{-18.521}$ & $<0.001$ \\
\hline IGRA, negative or not done & 1.782 & $0.993-3.199$ & 0.053 & & & \\
\hline
\end{tabular}

TB, tuberculosis; OR, odds ratio; CI, confidence interval; PCR, polymerase chain reaction; IGRA, interferon-gamma release assay.

should consider the possibility of TB in elderly patients living in areas with a considerable TB burden.

In our study, BMI was significantly higher in the missed TB group than in the control group. We, therefore, assumed that clinicians may be less likely to suspect TB in patients with higher BMI, although there is limited in-depth evidence to support this assumption. Although there were significant differences in BMI in the two groups, the mean BMIs in both groups were within the normal weight range according to the WHO guidelines.

According to the Korean TB guidelines [8], broad-spectrum antibiotics-except fluoroquinolones-could be used to rule out pulmonary TB in clinical settings. However, in our study, only $38 \%$ of patients who had a possible diagnosis of pulmonary TB were treated with broad-spectrum antibiotics before their diagnosis. This might be ex- plained by the high performance rate of chest CT $(98 \%$ in our study) and easy accessibility of bronchoscopy at our institution. At our facility, when a clinician suspects pulmonary TB through chest radiographs, they tend to perform chest CT and bronchoscopic examinations to achieve an accurate diagnosis, rather than initiate a trial of broad-spectrum antibiotics in our situation.

Rapid molecular diagnostic tools have improved the diagnostic yield of TB. Our analysis revealed that positive PCR results were associated with a faster diagnosis. However, we also found that the sensitivity of PCR testing was only $47.8 \%$. This was probably related to the properties of smear-negative TB, i.e., paucibacillary TB with low bacillary load or enzymes in the sputum, which inhibits the amplification reaction. Previous studies reported that Xpert MTB/RIF results had a sensitivity ranging from $47 \%$ to $87 \%$ [19] in the setting of 
smear-negative pulmonary TB. However, in real-world practice, the sensitivity of Xpert for the detection of TB is inadequate when few bacilli are present in the clinical specimen. Therefore, various attempts have been made to increase the sensitivity of the diagnosis. Although a recent report showed that the sensitivity of the Xpert MTB/RIF Ultra assay (63\%) was superior to that of the Xpert MTB RIF assay (46\%), neither test's sensitivity was sufficiently high in the setting of smear-negative pulmonary TB [20]. Thus, the development of further rapid and sensitive diagnostic tools for paucibacillary TB is warranted to prevent the delayed diagnosis of possibly infectious cases of pulmonary TB.

This study had some limitations. First, our study was a single-center trial, and the results may not be applicable to other settings. Second, the retrospective design of this study was limited to the review of medical records and our data may, therefore, not be as accurate as data collected within the context of a prospective study. Thus, further prospective studies are required to confirm our results.

In conclusion, we identified clinical characteristics and risk factors associated with missed TB diagnosis in patients who initiated anti-TB treatment after receiving confirmed positive culture results. These factors could provide clues and explain why the diagnosis of TB was delayed in the missed TB group. Carefully considering the possibility of TB in an elderly population, even when symptoms are atypical, and performing diagnostic tests-including radiological imaging and molecular assays-can help clinicians with the early detection of TB.

\section{KEY MESSAGE}

1. Patients with a delayed diagnosis of tuberculosis (TB) were older, had a higher body mass index, and were more likely to have negative polymerase chain reaction results than those in whom empirical treatment was initiated before positive cultures.

2. The development of further rapid and sensitive diagnostic tools for paucibacillary TB is necessary to prevent the delayed diagnosis of pulmonary TB.

\section{Conflict of interest}

No potential conflict of interest relevant to this article was reported.

\section{REFERENCES}

1. World Health Organization. WHO Guidelines on Tuberculosis Infection Prevention and Control, 2019 Update. Geneva (CH): World Health Organization, 2019.

2. Churchyard G, Kim P, Shah NS, et al. What we know about tuberculosis transmission: an overview. J Infect Dis 2017;216:S629-S635.

3. Rossato Silva D, Muller AM, Dalcin Pde T. Factors associated with delayed diagnosis of tuberculosis in hospitalized patients in a high TB and HIV burden setting: a cross-sectional study. BMC Infect Dis 2012;12:57.

4. Piatek AS, Van Cleeff M, Alexander H, et al. GeneXpert for TB diagnosis: planned and purposeful implementation. Glob Health Sci Pract 2013;1:18-23.

5. Aber VR, Allen BW, Mitchison DA, Ayuma P, Edwards EA, Keyes AB. Quality control in tuberculosis bacteriology. 1. Laboratory studies on isolated positive cultures and the efficiency of direct smear examination. Tubercle 1980;61:123-133.

6. Korea Centers for Disease Control and Prevention; Korea National Institute of Health; Korean Society of Clinical Micorbiology. Manual of Laboratory Tests for Tuberculosis. Cheongju (KR): Korea Centers for Disease Control and Prevention, 2013.

7. World Health Organization. Guidelines for Treatment of Tuberculosis. 4th ed. Geneva (CH): World Health Organization, 2010.

8. Korea Academy of Tuberculosis and Respiratory Diseases; Korea Centers for Disease Control and Prevention. Korean Guidelines for Tuberculosis. 3rd ed. Cheongju (KR): Korea Centers for Disease Control and Prevention, 2017.

9. World Health Organization. Definitions and Reporting Framework for Tuberculosis: 2013 Revision. Geneva (CH): World Health Organization, 2013.

10. Heo DH, Seo JW, Kim JH, et al. Delays in isolating patients admitted to hospital with pulmonary tuberculosis in Korea. J Korean Med Sci 2019;34:e270.

11. Storla DG, Yimer S, Bjune GA. A systematic review of delay in the diagnosis and treatment of tuberculosis. BMC Public Health 2008;8:15. 
12. Lee CH, Wang JY, Lin HC, et al. Treatment delay and fatal outcomes of pulmonary tuberculosis in advanced age: a retrospective nationwide cohort study. BMC Infect Dis 2017;17:449.

13. Yoshikawa TT. Tuberculosis in aging adults. J Am Geriatr Soc 1992;40:178-187.

14. Lee $\mathrm{SH}$. Active case finding in the elderly tuberculosis in South Korea. Tuberc Respir Dis (Seoul) 2019;82:261-263.

15. Cantalice Filho JP, Boia MN, Sant Anna CC. Analysis of the treatment of pulmonary tuberculosis in elderly patients at a university hospital in Rio De Janeiro, Brazil. J Bras Pneumol 2007;33:691-698.

16. Shu CC, Lee CH, Lee MC, Wang JY, Yu CJ, Lee LN. Hepatotoxicity due to first-line anti-tuberculosis drugs: a fiveyear experience in a Taiwan medical centre. Int J Tuberc
Lung Dis 2013;17:934-939.

17. Leung CC, Yew WW, Chan CK, et al. Tuberculosis in older people: a retrospective and comparative study from Hong Kong. J Am Geriatr Soc 2002;50:1219-1226.

18. Han J, Nam BD, Park SY, et al. Risk factors for delayed isolation of patients with active pulmonary tuberculosis in an acute-care hospital. Sci Rep 2019;9:4849.

19. Lombardi G, Di Gregori V, Girometti N, Tadolini M, Bisognin F, Dal Monte P. Diagnosis of smear-negative tuberculosis is greatly improved by Xpert MTB/RIF. PLoS One 2017;12:e0176186.

20. Dorman SE, Schumacher SG, Alland D, et al. Xpert MTB/ RIF Ultra for detection of Mycobacterium tuberculosis and rifampicin resistance: a prospective multicentre diagnostic accuracy study. Lancet Infect Dis 2018;18:76-84. 